\title{
Geneticists seek keys to bat vocalizations
}

\section{Bat $1 K$ project will sequence DNA of more than 1,000 species.}

\section{BY RAMIN SKIBBA IN SAN DIEGO, CALIFORNIA}

ome bats sing just as birds and humans do.

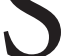
But how they learn their calls and melodies is a mystery - one that scientists will try to solve by sequencing the genomes of more than 1,000 bat species.

The project, called Bat $1 \mathrm{~K}$, was announced on 14 November at the annual meeting of the Society for Neuroscience in San Diego, California. Its organizers also hope to learn more about the flying mammals' ability to navigate in the dark through echolocation; their strong immune systems that can shrug off Ebola; and their relatively long lifespans.

"The genomes of all these other species, like birds and mice, are well-understood," says Sonja Vernes, a neurogeneticist at the
Max Planck Institute for Psycholinguistics in Nijmegen, the Netherlands, and co-director of the project. "But we don't know anything about bat genes yet."

Some bats show babbling behaviour, including barks, chatter, screeches, whistles and trills, says Mirjam Knörnschild, a behavioural ecologist at the Free University of Berlin. Young bats learn the songs and other sounds from older male tutors. They use these sounds during courtship and mating, when they retrieve food and as they defend their territory against rivals.

Scientists have studied the vocal sounds of only about 50 bat species so far, Knörnschild says, and they know much less about bats' communication than birds'. Four species of bat have so far been found to learn vocal sounds from each other, their fathers and other adult males, just as a child gradually learns how to speak from its parents (S. C. Vernes Psychon. Bull. Rev. http://doi.org/btcf; 2016).

\section{SINGING LESSONS}

Genetic studies have identified at least one gene in bats that is linked to speech and language, called FOXP2 (M. Knörnschild Curr. Opin. Neurobiol. 28, 80-85; 2014). The gene is also known to have a role in how people learn language, and in vocal learning in songbirds. Researchers working on the Bat $1 \mathrm{~K}$ project expect to find that other genes are also involved in communication, and that many more bat species have the ability to learn songs, calls and other sounds. "It's not a rare trait," Knörnschild says. "I'm becoming convinced that there's a whole continuum in bat vocal learning."

Bats' echolocation ability has been studied for many years, partly because of its applications to sonar and radar. But scientists know very little about the acoustic communication and social behaviour that drive how bats learn their songs and sounds, says Michael Yartsev, a neurobiologist at University of California, Berkeley. The study of vocal learning in bats is "nearly completely untapped", he says - likening it to the state of research into birdsong 60 years ago. 